\title{
STUDY ON BIODEGRADABLE AND ANTIMICROBIAL PROPERTIES OF BIOWASTE CHITIN-POLYVINYL ALCOHOL BLENDED FILM
}

\author{
Aung Than Htwe, Maung Htwe, Soe Maung, and Myint Naing Tun \\ Department of Chemistry, University of Yangon, Yangon, Myanmar, \\ Tel: +959957309102, e-mail: aungthanhtwe76@gmail.com, aungthanhtwe@uy.edu.mm
}

Received Date: December 26, 2019; Revised Date: March 14, 2021; Acceptance Date: April 7, 2021

\begin{abstract}
The film forming ability of chitin (CT) blended with polyvinyl alcohol (PVA) were prepared with a series of nine different ratios of CT:PVA, (10:90, 20:80, 30:70, 40:60, 50:50, 60:40, 70:30, 80:20, 90:10v/v), using $2 \%$ chitin solution and $10 \%$ polyvinyl alcohol solution. All prepared blended films have a highly transparent, smooth surface and pale-yellow color. The prepared blended films were characterized by using the physical parameters, the physico-mechanical properties, the degree of swelling, and water uptake. As measured by the swelling and water uptake, the blended films showed a higher degree of hydration, as a result of varying the weight percent of PVA in the membrane matrix. Comparative characterization of the prepared blended films included FT IR and TG-DTA analysis. From the FT IR analysis, the characteristic absorption peaks of CT-PVA blended film clearly showed that the two polymers were well mixed. According to TG-DTA analysis, the thermal stability of the CT-PVA blended film was found to be slightly lower. The various types of CT-PVA blended films were also tested for antimicrobial activity using an agar disc diffusion method. From these results, all of the prepared CT-PVA blended film showed pronounce antimicrobial activities. Subsequently, the biodegradable nature of the prepared CT-PVA blended films was studied through the soil burial test. Finally, the prepared CT-PVA blended films can be used in wound dressing, and also as packaging materials.
\end{abstract}

Keywords: Antimicrobial activity, Biodegradable nature, Chitin, Physicomechanical properties, Polyvinyl alcohol

\section{Introduction}

Non-biodegradable polymers cause the increase of worldwide environmental pollution. The research on biodegradable polymers is both necessary and valuable, supporting global sustainability and helping to solve the petroleum crisis and environmental problems. However, the poor solubility in most organic solvents restricts the utilization [1]. Chitin or poly ( $N$-acetylD-glucosamine) is a polysaccharide, which is abundantly available in nature as a component in cell walls of various fungi, as well as in shells of various insects and crustaceans. Chitin is predominantly present as a fibrillose crystalline material. Based on infrared spectroscopy and $\mathrm{X}$-ray diffraction data, chitin can be found in one of the three crystalline forms: $\alpha$-chitin, $\beta$ chitin and $\gamma$-chitin. The molecules in orthorhombic $\alpha$-chitin are arranged very tightly in an antiparallel fashion. $\alpha$-Chitin is mainly present in shells of crabs, lobsters and shrimps. $\beta$-Chitin, obtained from squid pens, takes the monoclinic form in which the chains arranged in a parallel fashion, while $\gamma$-chitin is the form in which the molecules are arranged in both parallel and antiparallel manner. As a result of the molecular packing, intermolecular interactions in $\beta$-chitin are 
weaker than those in $\alpha$-chitin, making $\beta$-chitin more susceptible to dissolution in a number of solvents. This finally results in $\beta$-chitin being more reactive and versatile [2]. Like several natural adsorbent, such as clay in tubular membrane; this polymer is biodegradable, biocompatible, and could assist in the reduction of pollutants in residual waters by chelating with heavy metallic ions, and absorbing the industrial dyes and pesticide. This biopolymer has a wide range of applications in the medical field, cosmetics, food industry, and especially in the wastewater treatment process [3]. Poly vinyl alcohol (PVA) is a nontoxic, water-soluble synthetic polymer that has good film-forming ability. It has a large number of hydroxyl groups, which allows it to react with many types of functional groups [4]. Polymer blending is one of the useful ways to have a new material with required properties, and there has been a great scientific and commercial progress in the area of polymer blends [5,6].

In this research, chitin/PVA blend films were prepared by solvent casting from solutions of chitin and PVA in concentrated formic acid at various compositional ratios. The effects of the blended compositions on physical properties, thermal properties, mechanical properties, morphology, and swelling behavior were studied.

\section{Materials and Methods}

\section{Materials}

Polyvinyl alcohol (Molecular weight 20,000, degree of hydrolysis 98\%) was purchased from the British Drug House (BDH) Chemical Ltd., England. All other chemicals used were of analytical reagent grade. In all investigations, the recommended standard methods and techniques involving both conventional and modern methods were provided.

\section{Preparation of Chitin-Polyvinyl Alcohol (CT-PVA) Blended Films}

Chitin solution $2 \% \mathrm{w} / \mathrm{v}$ was prepared by dissolving $2 \mathrm{~g}$ of chitin (CT) in $100 \mathrm{~cm}^{3}$ of $90 \% \mathrm{v} / \mathrm{v}$ formic acid with frequent stirring for 30 minutes at room temperature to get a clear solution. A clear solution of PVA $10 \% \mathrm{w} / \mathrm{v}$ was placed in autoclave for 20 minutes at $120^{\circ} \mathrm{C}$ and $0.1 \mathrm{MPa}$ in water. The prepared CT solution and PVA solution were mixed with appropriate proportions to obtain a series of CT-PVA blends. The blends were prepared in nine different ratio of CT: PVA, (10:90, 20:80, 30:70, 40:60, 50:50, 60:40,70:30, 80:20, 90:10 v/v) by stirring thoroughly the mixed polymer solution for about 30 minutes. The blended polymer solutions were kept for a period of time to remove any bubble formation and were subsequently casted onto a clean and dried melamine plate at room temperature to form a membrane-like. The melamine plates containing the blended solution were kept for about 3 days to obtain CT-PVA blended films. After drying for 3 days, the membranes were easily removed from the melamine plates. The membranes were immersed in $1 \mathrm{M} \mathrm{NaOH}$ solution to remove residual materials, and were then washed with distilled water to remove the alkali and unreacted materials. This was followed by the drying at room temperature for 3 days.

\section{Physical Parameter Measurement of CT-PVA Blended Solution}

\section{Determination of $p H$}

Each of the sample solution was placed into separate beakers. The $\mathrm{pH}$ of the solution was measured by using $\mathrm{pH}$ meter at room temperature. 


\section{Determination of Specific Gravity}

A SG density bottle $\left(50 \mathrm{~cm}^{3}\right)$ was washed and cleaned with distilled water, thoroughly dried and subsequently weighed. Then the SG bottle was filled with distilled water at room temperature. The stopper was firmly inserted in the bottle, and excess distilled water exiting from the capillary of the stopper was wiped carefully with a piece of tissue paper, then the bottle containing water was weighed. In order to determine the specific gravity of the sample, the bottle was thoroughly dried and filled with the different CT-PVA solution at room temperature. The excess solution from the capacity was wiped with the piece of tissue paper and weighed. The specific gravity of different samples was calculated using the following Equation (1) [8].

$$
\text { Specific gravity }(\mathrm{SG})=\frac{\mathrm{W}_{3}-\mathrm{W}_{1}}{\mathrm{~W}_{2}-\mathrm{W}_{1}} \times \text { density of liquid }
$$

Where, $\mathrm{W}_{1}$ is the weight of the density bottle in grams, $\mathrm{W}_{2}$ is the total weight of the density bottle with distilled water in grams and $\mathrm{W}_{3}$ is the total weight of the density bottle with sample solution in grams.

\section{Determination of Viscosity}

About $50 \mathrm{~cm}^{3}$ of different sample solution was placed into the viscometer cup. The solution was kept in a constant temperature bath at $30^{\circ} \mathrm{C}$ and was measured the rotational viscosity (cPs) with VISCO viscometer [7]. A VISCO 6800-E07 viscometer, ATAGO, Japan was used for determining the viscosity.

\section{Determination of Physicomechanical Properties}

The tensile strength, elongation at break and tear strength of the samples were measured on the Tensile testing machine (Hourns field 5000E), Cutter (Wallace) at room temperature with the rate of moveable jaw $100 \mathrm{~mm} / \mathrm{min}$ according to JIS K 7127 (1987). Three samples were measured and average values were reported.

\section{Determination of Water Uptake (\%)}

The films to be tested were cut into $1 " \times 1$ " size. The cut films were immersed in distilled water at room temperature for the specified period of time. The films were removed from distilled water in beaker, blotted gently with tissue paper, weighed, and then put back into beaker for the next measurement. The water uptake of different samples was calculated using the Equation (2) [8].

$$
\text { Water uptake }(\%)=100 \times \frac{\mathrm{W}_{2}-\mathrm{W}_{1}}{\mathrm{~W}_{1}}
$$

Where, $\mathrm{W}_{1}$ is the weight of completely dried sample and $\mathrm{W}_{2}$ is the weight of swelled sample in the distilled water at room temperature for 1 hour.

\section{Determination of Degree of Swelling}

The films to be tested were cut into $1 " \times 1$ " size. The films were immersed in distilled water at room temperature for the specified period of time. The films were removed from distilled 
water in beaker, blotted gently with tissue paper, weighed, and then put back into the beaker for the next measurement. The procedure was continued until no more water is absorbed. Based on these values, swelling (\%) were determined. Each experiment was replicated three times. The degree of swelling was determined according to Equation (3).

$$
\text { Degree of swelling }(\%)=\frac{\mathrm{W}_{\mathrm{s}}-\mathrm{W}_{\mathrm{d}}}{\mathrm{W}_{\mathrm{s}}} \times 100
$$

Where, $\mathrm{W}_{\mathrm{s}}$ and $\mathrm{W}_{\mathrm{d}}$ represent the weight of the films after and prior to immersion. All the experiments were carried at room temperature.

\section{Identification and Characterization of Selective CT-PVA Blended Films}

The prepared blended films were analyzed by FTIR in a wide range wavelength between 400 $\mathrm{cm}^{-1}$ and $4000 \mathrm{~cm}^{-1}$ with resolution of $1 \mathrm{~cm}^{-1}$ and $3 \mathrm{scans} /$ sample. A Perkin Elmer GX System FT-IR spectrophotometer was used. Thermogravimetric analysis was carried out in nitrogen atmosphere at a heating rate of $20.0 \mathrm{kJmin}^{-1}$ and scanning from $40^{\circ} \mathrm{C}$ to $600^{\circ} \mathrm{C}$. TG-DTA thermoanalyser (Model BAHR-Thermoanalyser GmbH, Germany) was used.

\section{Determination of Antimicrobial Test}

The different CT-PVA blended films were tested with Bacillus subtilis, staphylococcus aureus, Pseudomonus aeruginosa, Bacillus pumalis, Candida albican and E. coli species to investigate the nature of antimicrobial activity. After preparing the bacteriological medias, the dried films were placed on the agar with flamed forceps and gently pressed down to ensure proper contact. The plates were incubated immediately or within $30 \mathrm{~min}$ after incubation. After overnight incubation at $37^{\circ} \mathrm{C}$, the results were recorded by photo.

\section{Determination of Biodegradation by Soil Burial Test}

Biodegradation of prepared films was determined by soil burial test to examine the weight loss and morphology changes. The films were cut into $2 " \times 2$ " dimensions. The films were then accurately weighed and buried in the soil at the depth of $5 \mathrm{~cm}$. They were taken out from the soil at an interval of one week. Sample geometry on degradation was also recorded by photo.

\section{Results and Discussion}

In the present contribution chitin-polyvinyl alcohol (CT-PVA) blended films were prepared by solution casting from solutions of chitin in dilute formic acid and polyvinyl alcohol in the distilled water at various compositional ratios. The effect of blended composition on physicochemical properties. Physicomechanical properties, water uptake, swelling behavior, thermal properties, Fourier transform infrared spectroscopy and biodegradable and antimicrobial properties were studied. Solution of CT-PVA blended solutions appeared to be homogenous. After evaporation of the solvent, the prepared CT-PVA blended films were found to be transparent.

\section{Physical Parameters of CT-PVA Solution}

Figure 1(a), (b) and (c) shows the physical parameters of $2 \%$ chitin, $10 \%$ PVA and different CT-PVA blended solutions. The measuring parameters were $\mathrm{pH}$, specific gravity and viscosity. From the value of $\mathrm{pH}$ it can be observed that $2 \%$ chitin, $1 \%$ PVA and different CT-PVA blended 
solutions are slightly acidic solutions in the range of 4.09 to 4.89 . The specific gravity of $2 \%$ chitin, 10\% PVA and different CT-PVA blended solutions are in the range of 1.032 to 1.357 . It can be seen that there is no significant changes in specific gravity. The viscosity of $2 \%$ chitin, 10\% PVA and different CT-PVA blended solutions are between 523 to $1.5 \mathrm{cPs}$. Thus, all physical parameters of CT-PVA blended solutions showed slightly different values.
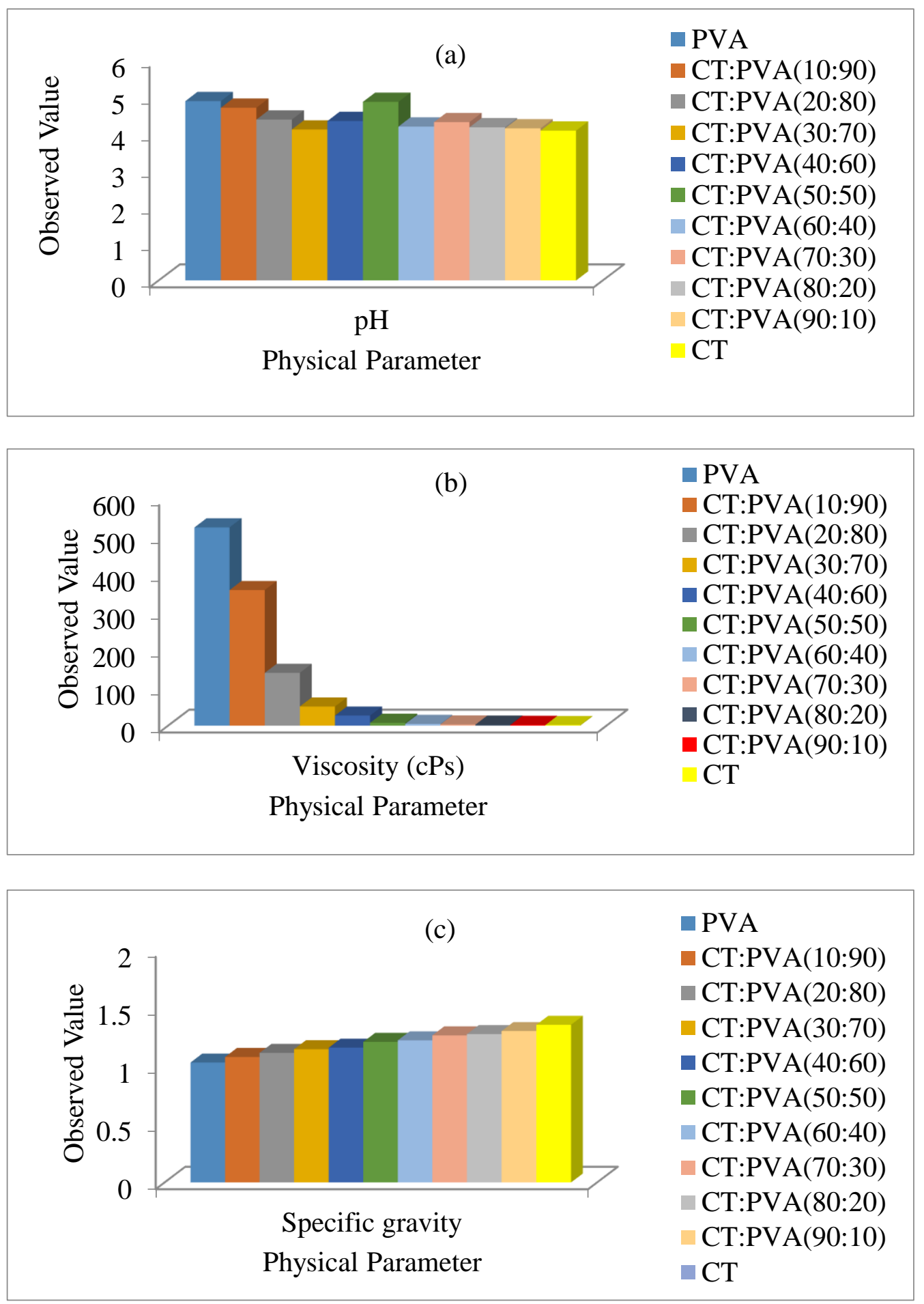

Figure 1. Physicochemical properties of CT-PVA solutions 


\section{On the Aspect of Characterization of CT-PVA Films}

The physicomechanical properties in terms of tensile strength, elongation at break (\%), and tear strength are important parameters which revealed the nature of films. The physicomechanical properties of CT-PVA blended films are presented in Figure 2. The thickness of CT- PVA hydrogel films are in the range of $0.10 \mathrm{~mm}$ to $0.60 \mathrm{~mm}$. Tensile strength decreased from $10.7 \% \mathrm{MPa}$ to $3.3 \% \mathrm{MPa}$. The present elongation at break drastically CTPVA (20:80) 335\% content, however, it was significantly increased at CT-PVA (70:30) 100\% to CT-PVA (20:80) 335\%. As seen in Figure 2, the tensile strength and elongation values percent are flexible in the range of CT-PVA (80:20) and CT-PVA (20:80). The tear strength is another mechanical property of the nature of the films. It indicated that the tear strength of CT-PVA blended films are significantly decreased from $73 \%$ for CT-PVA (80:20) to $15 \%$ for CT-PVA (60:40). It can be concluded that according to the physicomechanical properties, such as tensile strength, elongation percentage at break, tear strength and thickness, the composition of CT-PVA (80:20), and CT-PVA (20:80) are flexible.

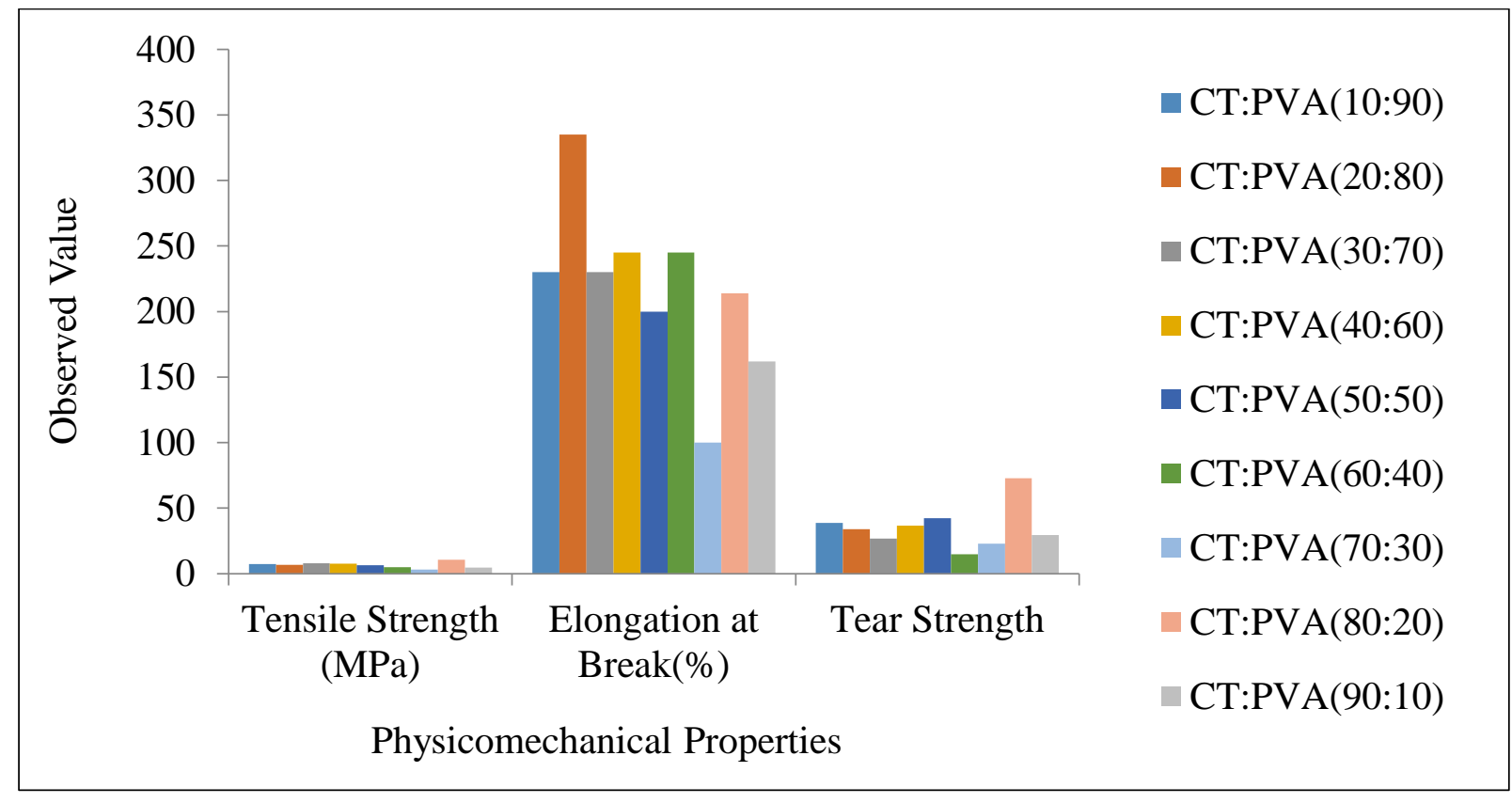

Figure 2. Physicomechanical properties of CT-PVA blended films

\section{Water Uptake (\%)}

The degree of water uptake was investigated with increasing immersion time. The water uptake as a function of time for CT-PVA blended film is shown in Figure 3. The water uptake is one of the most significant parameter when a film is to be used as biodegradable materials. The water uptake is the amount of water entrapped in the matrix including bound water. Figure 3 shows the water uptake percent for CT-PVA blended films. The water uptake of CT-PVA blended film was significantly different according to the various ratios CT-PVA blended films. CT-PVA (80:20) had the highest degree of water uptake content $(904.5 \%)$ at 30 minutes. The water uptake degree of every CT-PVA blended film increased with increasing time. The water uptake may impart stickiness and durability. 


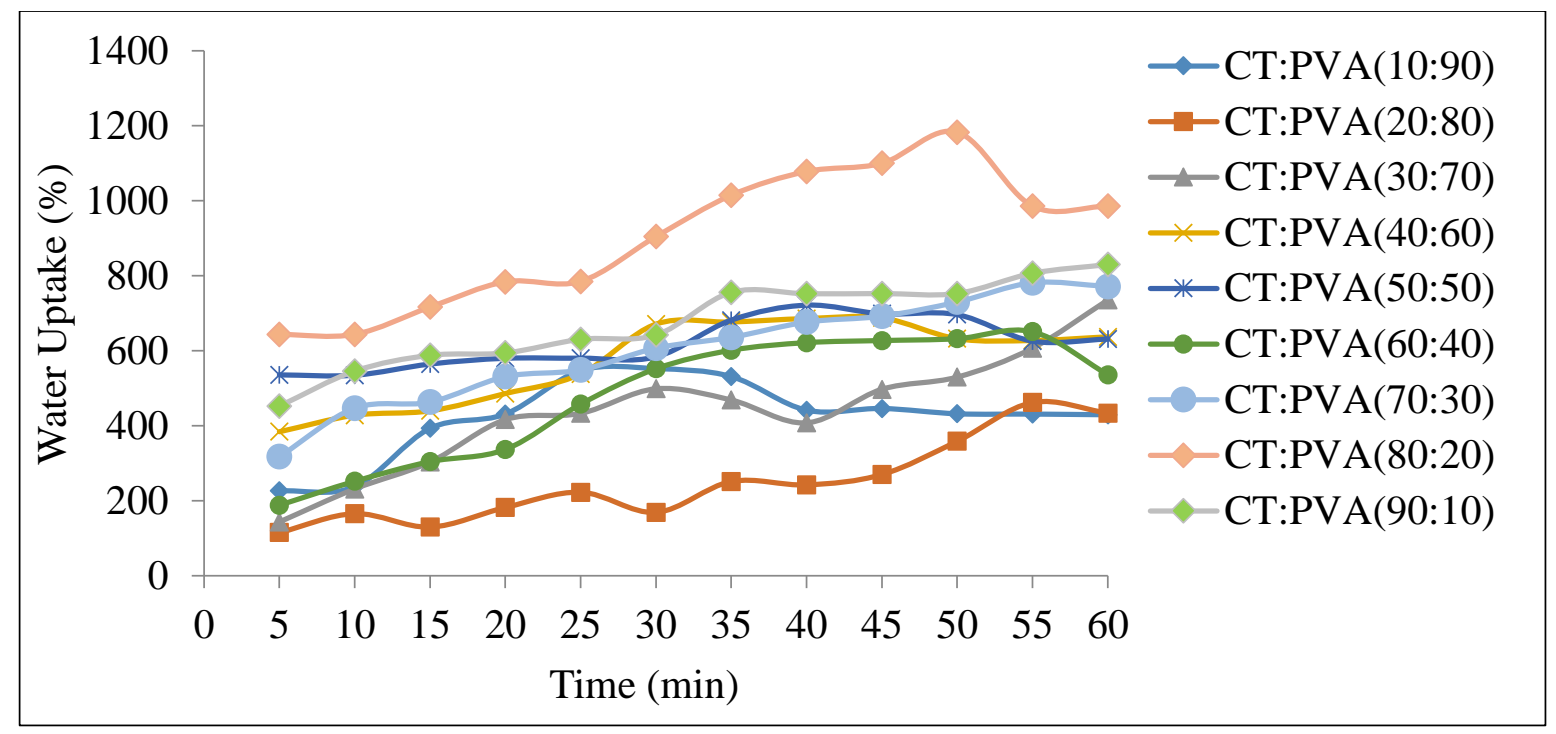

Figure 3. Degree of water uptake percent of CT-PVA as a function of contact time

\section{Aspect of Degree of Swelling (\%)}

The degree of swelling of CT-PVA blended films is shown in Figure 4 as a function of immersion time in distilled water at room temperature. For a given blended film composition, the degree of swelling mostly increased with increasing immersion time. It can also be observed that the degree of swelling increased with increasing PVA content in the blended film. The maximum value for the degree of swelling of CT-PVA (80:20) blended film was $90.04 \%$ at 30 minutes.

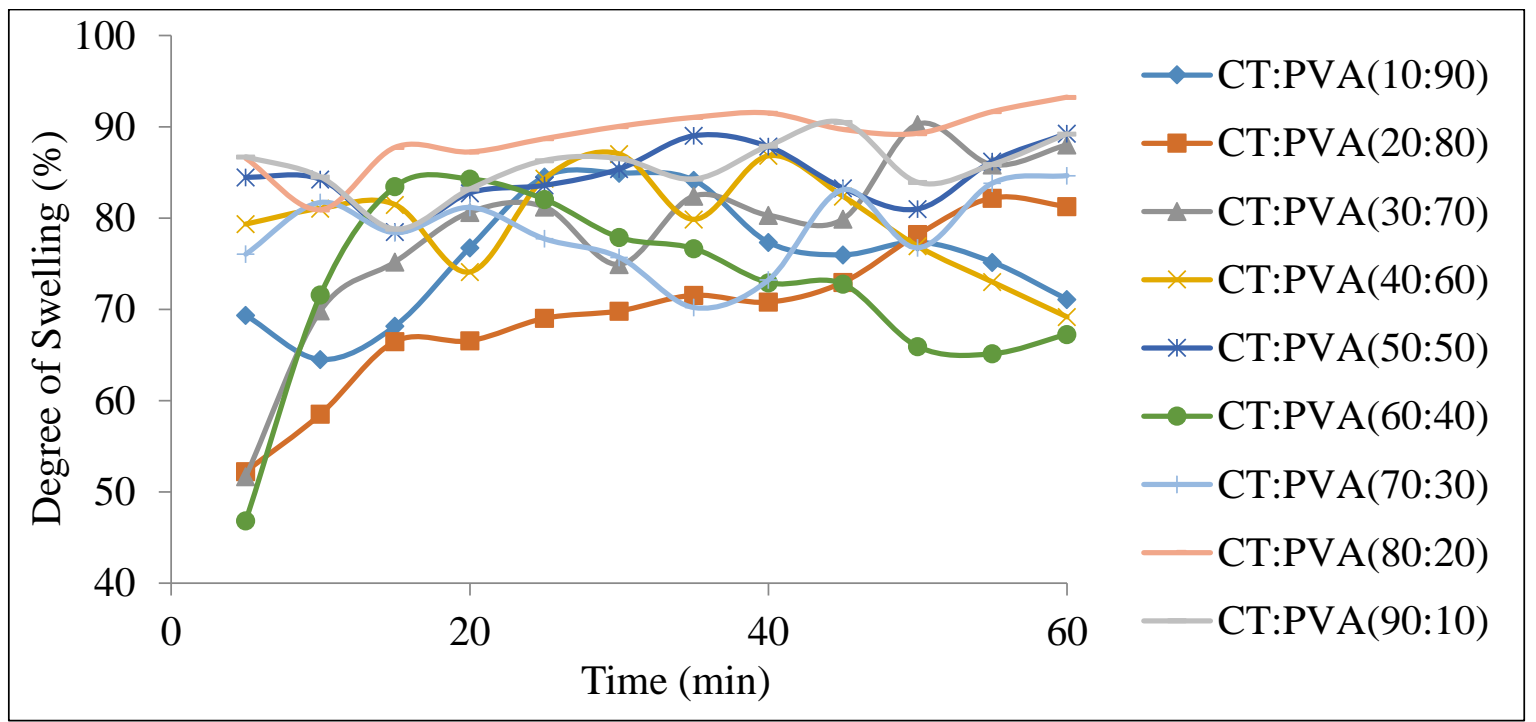

Figure 4. Degree of swelling percent of CT-PVA as a function of contact time.

\section{Characterization}

In this study, the blended samples of CT-PVA of 20:80, 40:60, 50:50, 60:40 and 80:20 volume ratio were chosen and characterized by FT IR and TG DTA analysis. After that antimicrobial properties and biodegradable nature of the chosen blend films were determined by agar plate diffusion method and soil burial method. 


\section{FT IR Analysis}

FTIR spectroscopic studies show the characteristics bands corresponding to vibrations of the hydroxyl-, methyl-, and methylene-, carbonyl-, and amide groups analyzed. It also provides data about the interactions between the polymeric components in the blends. The comparison of FTIR spectra of films such as CT-PVA (20:80), CT-PVA (40:60), CT-PVA (50:50), CTPVA (60:40) and CT-PVA (80:20) films are presented in Figure 5 and Table 1.

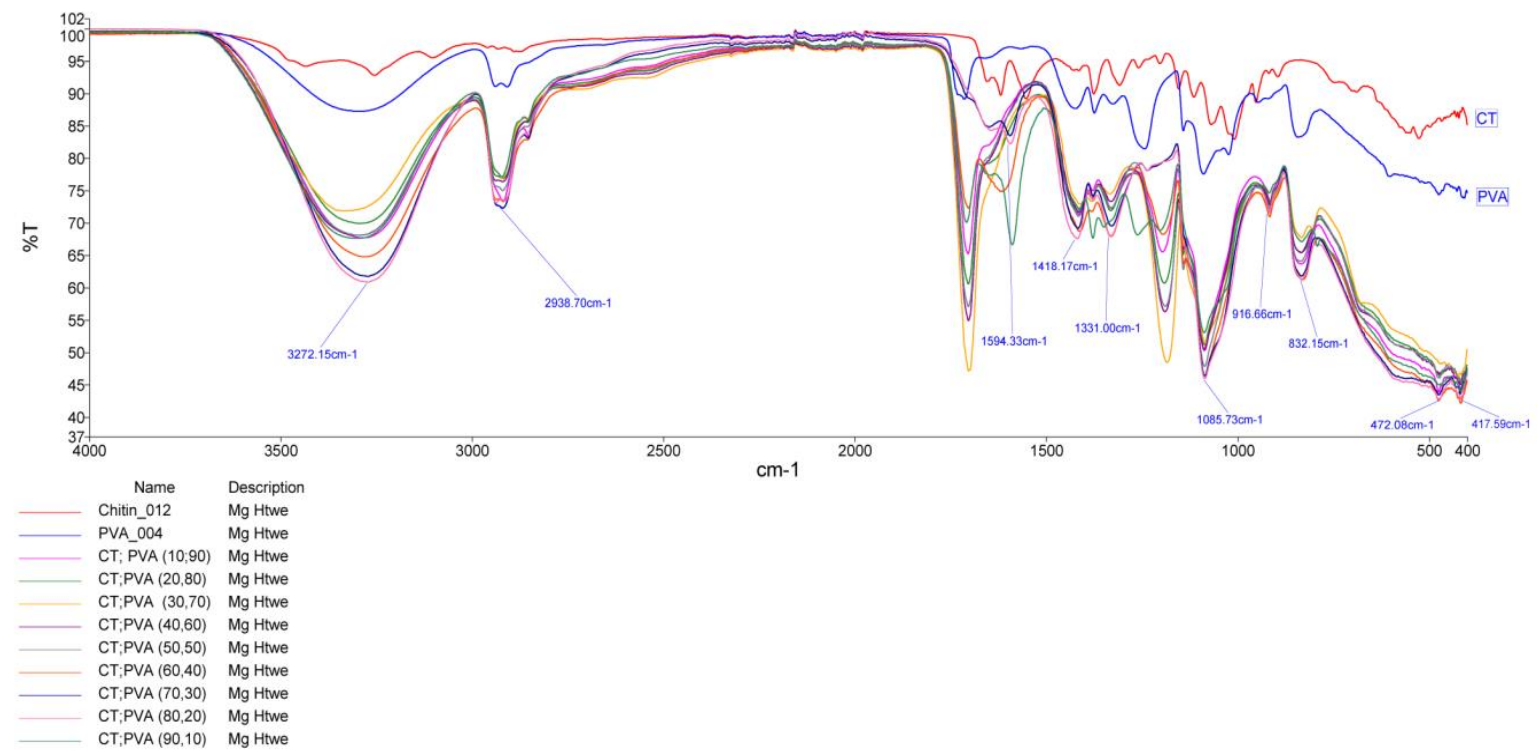

Figure 5. FT IR Spectral data for chitin-polyvinyl alcohol (CT-PVA) blended films

Table 1. FT IR Characteristic Bands of CT-PVA Blended Films [9, 10]

\begin{tabular}{ccccccccc}
\hline CT:PVA & $\begin{array}{c}\text { C-C \& } \\
\text { C-H } \\
\text { bend }\end{array}$ & C-H & $\begin{array}{c}\text { C-OH } \\
\text { stretch }\end{array}$ & CH & $\begin{array}{c}\text { NH } \\
\text { amide } \\
\text { I }\end{array}$ & $\begin{array}{c}\text { C=O } \\
\text { amide } \\
\text { I }\end{array}$ & $\begin{array}{c}\text { CH } \\
\text { stretch }\end{array}$ & $\begin{array}{c}\text { OH } \\
\text { stretch }\end{array}$ \\
\hline $100: 0$ & 896 & 952 & 1008 & 1331 & 1552 & - & 2874 & 3255 \\
$80: 20$ & 832 & 916 & 1085 & 1331 & 1418 & - & 2918 & 3272 \\
$60: 40$ & 831 & 916 & 1085 & 1330 & 1412 & 1703 & 2918 & 3282 \\
$50: 50$ & 835 & 916 & 1086 & 1330 & 1416 & 1703 & 2919 & 3294 \\
$40: 60$ & 834 & 915 & 1086 & 1330 & 1416 & 1703 & 2919 & 3305 \\
$20: 80$ & 833 & 915 & 1087 & 1331 & 1414 & 1703 & 2918 & 3295 \\
$0: 100$ & 842 & - & 1088 & - & 1424 & 1714 & 2909 & 3294 \\
\hline
\end{tabular}


As can be observed, the increase in the PVA concentration in the blended films caused a decrease in the intensity of the band arising from NH bending (amide II) at 1552 $\mathrm{cm}^{-1}$ of chitin. Also, an increase in the intensity of $\mathrm{CH}$ group at around $2938 \mathrm{~cm}^{-1}$ was found as the PVA content increases. As shown in the figure, all of the blended films agree to the ranges of absorption peaks and references peaks.

\section{Thermal Behavior from TG-DTA Analysis}

According to the TG-DTA thermogram profiles, the thermogram of CT-PVA $(20: 80)$, (40:60), (50:50), (60:40) and (80:20) blended films is shown in Figure 6. CT-PVA (20:80) blended film showed weight loss in three stages. The first stage is between the temperature ranges of about $39^{\circ} \mathrm{C}$ to $120^{\circ} \mathrm{C}$ with the absence of weight loss. There is no absorbed water and moisture. The second stage, the temperature range between $120^{\circ} \mathrm{C}$ to $410^{\circ} \mathrm{C}$ was observed with $71.24 \%$ of weight loss. This is due to the evaporation of water together with partial decomposition of chitin and PVA polymer. The third stage, $27.58 \%$ of weight loss was observed within the temperature range of $410^{\circ} \mathrm{C}$ to $600^{\circ} \mathrm{C}$. In this stage, weight loss is due to the complete degradation of chitin and PVA polymer.

Similarly, CT-PVA (40:60) blended film describes a weight loss in three stages. The first stage ranges between $38^{\circ} \mathrm{C}$ to $120^{\circ} \mathrm{C}$ with the absence of weight loss. There is no absorbed water and moisture. The second stage ranges between $120^{\circ} \mathrm{C}$ to $330^{\circ} \mathrm{C}$ with weight loss of $59.21 \%$. This weight loss is due to the decomposition of chitin and PVA polymer. The third stage ranges between $330^{\circ} \mathrm{C}$ to $600^{\circ} \mathrm{C}$ with weight loss of $37.59 \%$. This weight loss is due to the complete degradation of chitin and PVA polymer.

Thermal stability of CT-PVA (50:50) blended film is similarly presented. At the temperature range between $37^{\circ} \mathrm{C}$ to $140^{\circ} \mathrm{C}$, about $13.2 \%$ of weight loss was observed in the first stage. This is due to loss of bound water. In the second stage, the temperature range between $140^{\circ} \mathrm{C}$ to $300^{\circ} \mathrm{C}$ showed $59.81 \%$ of weight loss. This is due to the partial decomposition of chitin and PVA polymer. In the third stage, the temperature range between $300^{\circ} \mathrm{C}$ to $600^{\circ} \mathrm{C}$, about $19.59 \%$ of weight loss was observed. This is due to the complete degradation of chitin and PVA polymer.

In CT-PVA (60:40) blended film, the first stage temperature ranges between $38^{\circ} \mathrm{C}$ to $120^{\circ} \mathrm{C}$, while $6.52 \%$ of weight loss was observed. This is due to the loss of bound water. The second stage, range between $120^{\circ} \mathrm{C}$ to $410^{\circ} \mathrm{C}$ with weight loss of $58.68 \%$. This weight loss is due to decomposition of chitin and PVA polymer. The third stage, range between $410^{\circ} \mathrm{C}$ to $600^{\circ} \mathrm{C}$ with weight loss of $29.21 \%$. This weight loss is due to the complete degradation of chitin and PVA polymer.

Thermal stability of CT-PVA (80:20) blended film showed the temperature range between $38^{\circ} \mathrm{C}$ to $140^{\circ} \mathrm{C}$ with $6.55 \%$ of weight loss observed in the first stage. This is due to loss of bound water. In the second stage, the temperature ranges between $140^{\circ} \mathrm{C}$ to $330^{\circ} \mathrm{C}$ and $26.2 \%$ of weight loss was observed. This is due to partial decomposition of chitin and PVA polymer. In the third stage, the temperature ranges between $330^{\circ} \mathrm{C}$ to $600^{\circ} \mathrm{C}$ with $19.8 \%$ of weight loss was observed. This is due to complete degradation of chitin and PVA polymer. 


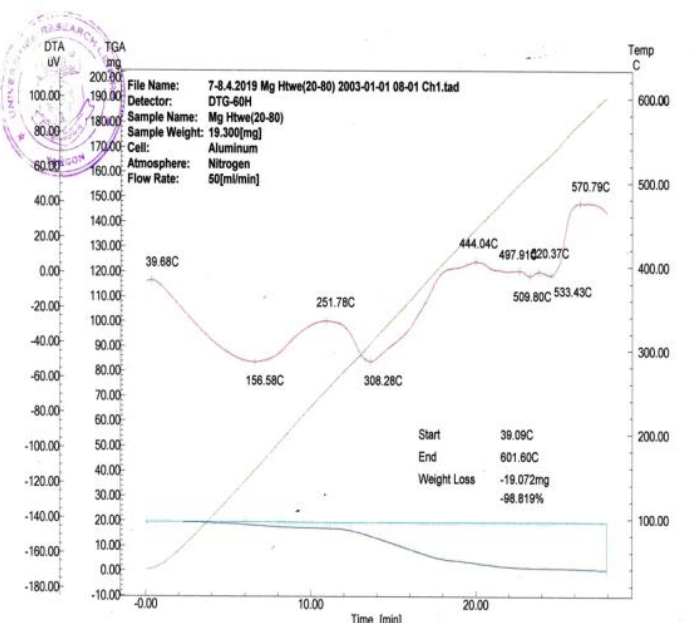

CT-PVA (20:80)

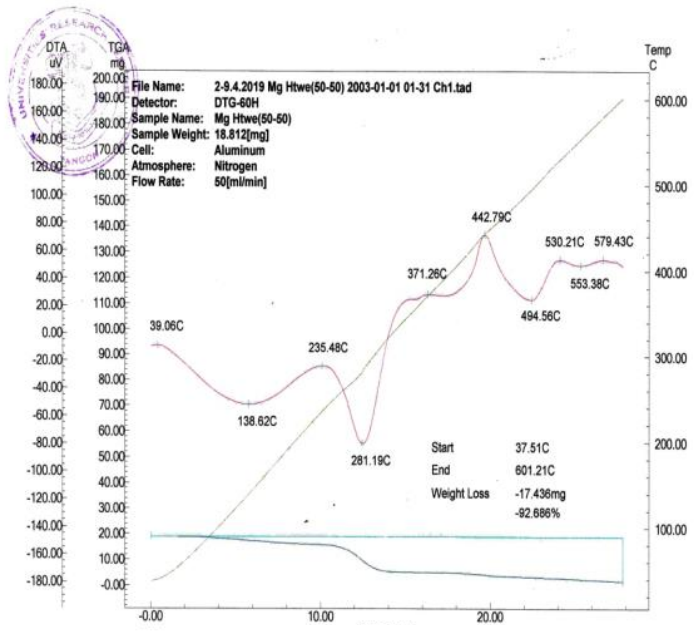

CT-PVA (50:50)

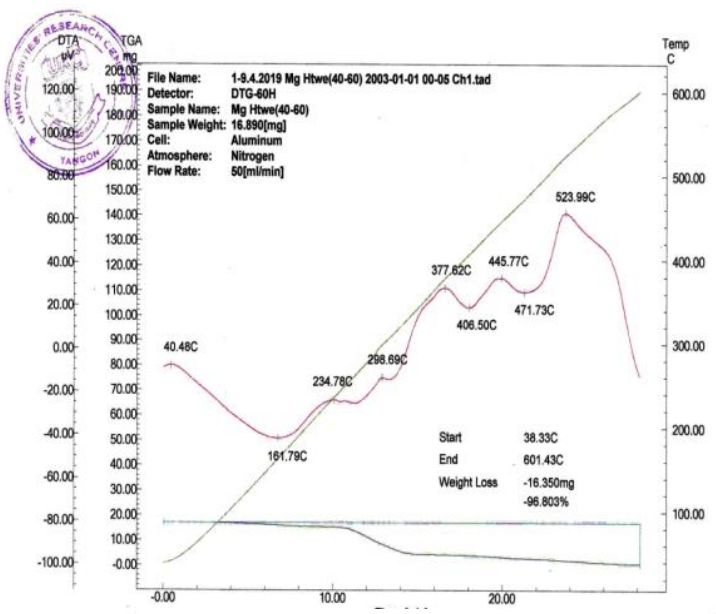

CT-PVA (40:60)

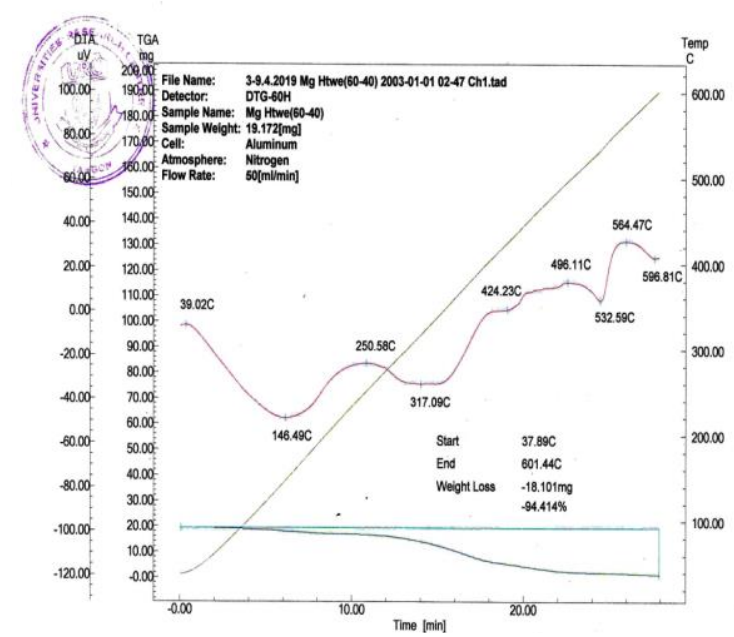

CT-PVA (60:40)

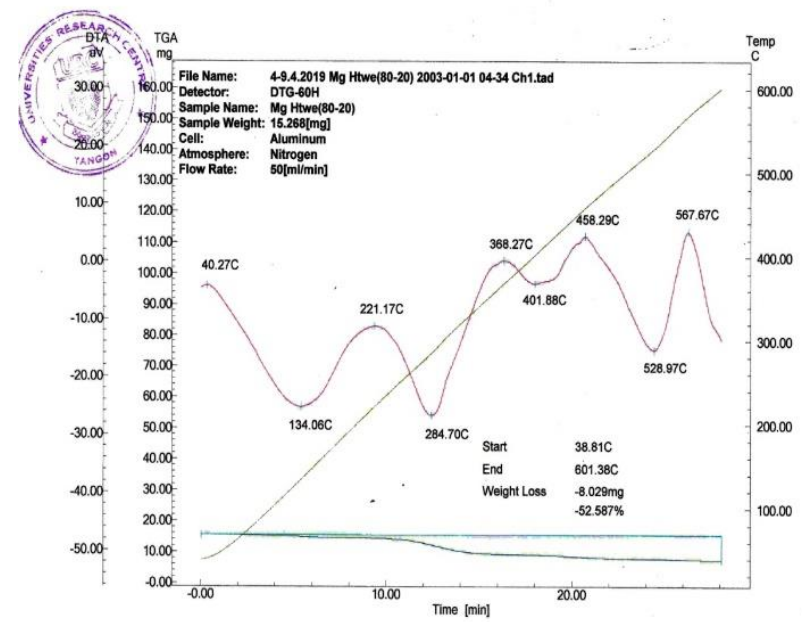

CT-PVA (80:20)

Figure 6. Thermal analysis data for chitin- polyvinyl alcohol (CT-PVA) blended film 


\section{Antimicrobial Activity of CT-PVA Blended Films}

Antimicrobial activity of CT-PVA (20:80), CT-PVA (40:60), CT-PVA (50:50), CT-PVA (60:40) and CT-PVA (80:20) blended films are shown in Table 2. The tested organisms were Bacillus subtilis, Staphylococcus aureus, Pseudomonas aeruginosa, Bacillus pumilus, Candida albican, and E.Coli. As indicated in Figure 7 and Table 2, antimicrobial test of CT-PVA (20:80), CT-PVA (40:60), CT-PVA (50:50), CT-PVA (60:40) and CT-PVA (80:20) blended films used the agar medium cultivation. As the results of the blended films, they all have antimicrobial activity. It can be concluded that according to the antimicrobial activity, the CT-PVA blended film may be used as burn dressing and food packaging materials.

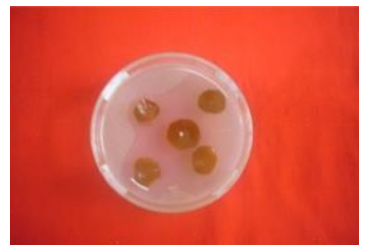

Bacillus subtilis

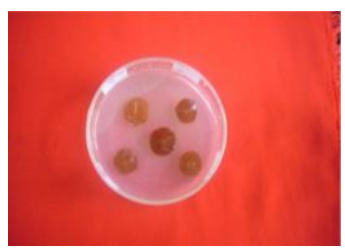

Candida albicans

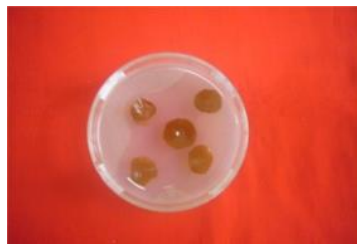

Staphylococcus aure

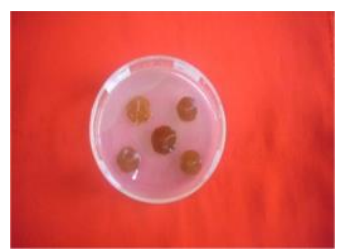

E. coli

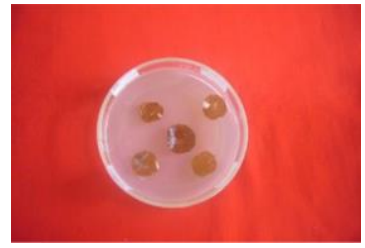

Pseudomonas aeruginosa

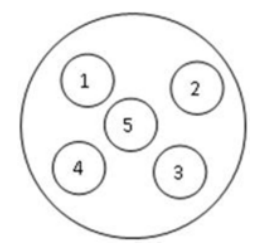

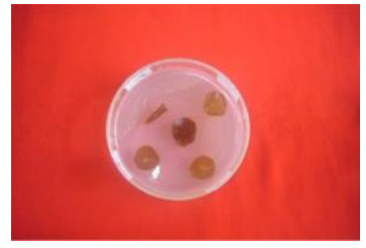

Bacillus pumilus

(1) CT-PVA(20:80)

(2) CT-PVA(40:60)

(3) CT:PVA(50:50)

(4) CT:PVA(60:40)

(5) $\mathrm{CT}: \operatorname{PVA}(80: 20)$

Figure 7. Antimicrobial activity of prepared CS-PVA blended film

Table 2. Antimicrobial Activity of CT-PVA Blended Film by Agar Disc Diffusion Method

\begin{tabular}{|c|c|c|c|c|c|c|}
\hline \multirow[b]{2}{*}{ No } & \multirow[b]{2}{*}{ Microbial } & \multicolumn{5}{|l|}{ Samples } \\
\hline & & $\begin{array}{l}\text { CT-PVA } \\
(20: 80)\end{array}$ & $\begin{array}{l}\text { CT-PVA } \\
(40: 60)\end{array}$ & $\begin{array}{l}\text { CS-PVA } \\
(50: 50)\end{array}$ & $\begin{array}{l}\text { CS-PVA } \\
(60: 40)\end{array}$ & $\begin{array}{l}\text { CS-PVA } \\
(80: 20)\end{array}$ \\
\hline 1 & Bacillus subtilis & + & + & + & + & + \\
\hline 2 & $\begin{array}{l}\text { Staphylococcus } \\
\text { aureus }\end{array}$ & + & + & + & + & + \\
\hline 3 & $\begin{array}{l}\text { Pseudomonas } \\
\text { aeruginosa }\end{array}$ & + & + & + & + & + \\
\hline 4 & Bacillus pumilus & + & + & + & + & + \\
\hline 5 & Candida albican & + & + & + & + & + \\
\hline 6 & E-coli & + & + & + & + & + \\
\hline & $\begin{array}{ll}+ & = \\
+++ & =\end{array}$ & $\begin{array}{l}\text { comm } \\
\text { significal }\end{array}$ & $\begin{array}{l}++ \\
-\end{array}$ & $\begin{array}{l}= \\
=\end{array}$ & $\begin{array}{l}\text { ainent } \\
\text { eaction }\end{array}$ & \\
\hline
\end{tabular}

\section{Biodegradation test by soil burial}

In this work, biodegradation of blended films such as CT-PVA (20:80), CT-PVA (40:60), CTPVA (50:50), CT-PVA (60:40) and CT-PVA (80:20) films were tested by soil burial method. 
As described above, environmental effects mentioned in this work are moisture and soil which may be favorable conditions for the microbial growth. Soil burial is a traditional method to test samples for degradation because of its similarity to actual condition of waste disposal. Uniformly sized samples were buried in the soil from waste disposal. The physical appearances of blended films after in the soil buried are shown in Figure 8.

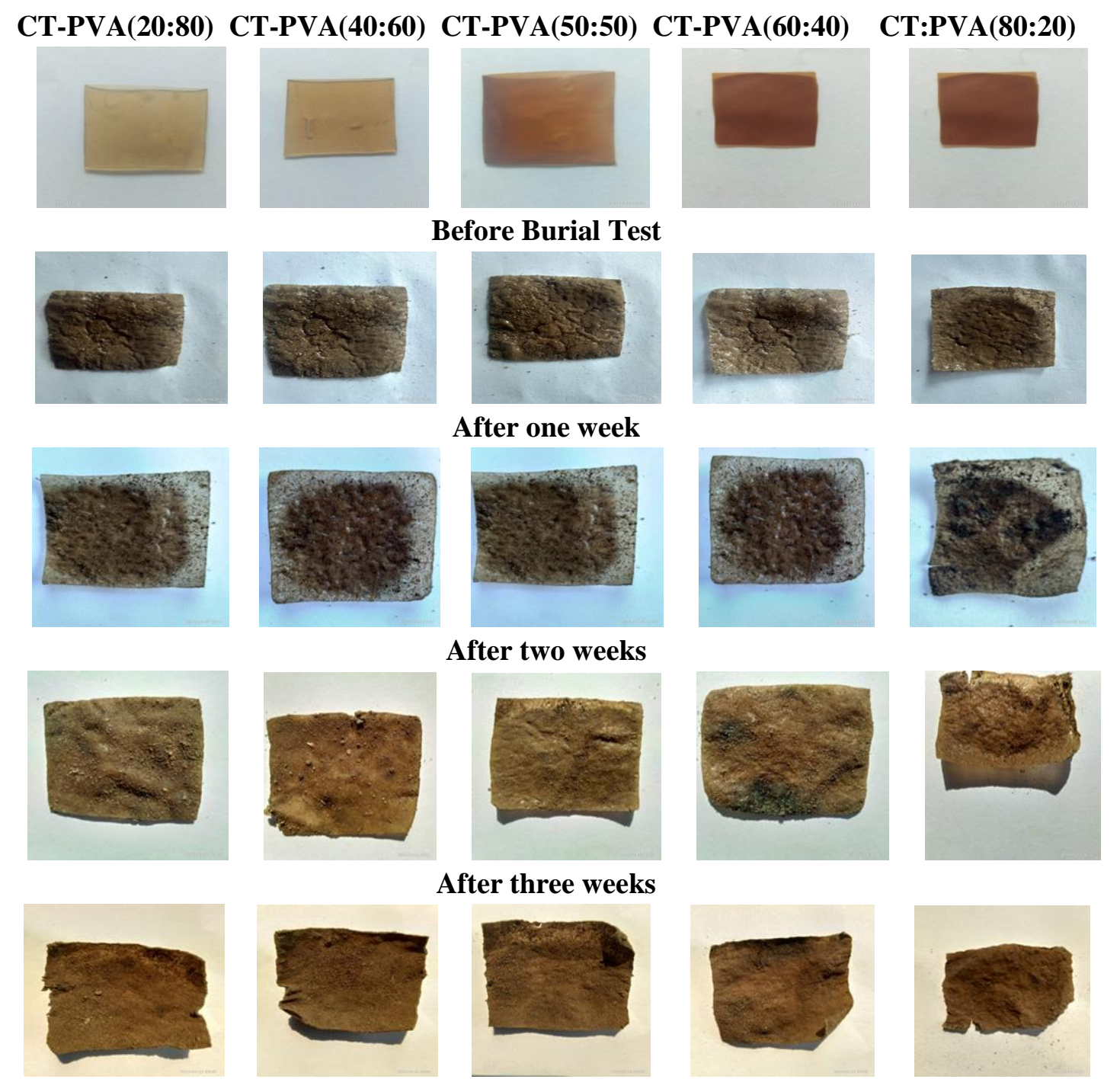

After four weeks

Figure 8 . The physical appearances of CT-PVA hydrogel Film by soil burial test.

Figure 8 shows biodegradation nature of CT-PVA (20:80), CT-PVA (40:60), CT-PVA (50:50), CT-PVA (60:40) and CT-PVA (80:20) blended films within one month interval. These figures clearly showed that a slight deformation of the blended films could be observed at each investigation period. By using the soil burial techniques, it was found that a slight deformation of all the CT-PVA blended film had occurred after 4 weeks.

\section{Conclusions}

Blended films of chitin and polyvinyl alcohol were prepared in various proportions. The prepared CT-PVA blended films show a clear, flexible, smooth, highly transparent, as well as 
light-yellow colored surface. The swelling and water uptake of blend membrane showed a higher degree of hydration, as measured by swelling and water uptake, which can be altered by varying the weight percentage of PVA in the membrane matrix. FT IR analysis indicated that there were existing intermolecular interactions between CT and PVA. From TG-DTA analysis, thermal stability of CT-PVA blended films was found to be slightly low. The various types of CT-PVA blended films were tested for its antimicrobial activities by using agar disc diffusion method. It can be noted that all of the microbial agents that came in contact with films were active. By using the soil burial techniques, it was found that a slight deformation of all the CTPVA blended films had occurred after 4 weeks. According to these results, the prepared CTPVA blended films could be used as the materials for burn wound dressing and food packaging.

\section{Acknowledgements}

The authors owe their gratitude to Professor Dr. Ni Ni Than, Head of Department, Department of Chemistry, University of Yangon, Yangon, Myanmar, for her stimulating suggestions.

\section{References}

[1] S. Zhang, F. Li, and J. Yu, "Preparation of cellulose/chitin blend biofibers via direct dissolution," Cellulose Chemistry and Technology, Vol. 43, No. 9-10, pp. 393-398, 2009.

[2] M. Peesan, R. Rujiravanit, and P. Supaphol, "Characterization of beta-chitin / poly (vinyl alcohol) blend films," Polymer Testing, Vol. 22, pp. 381-387, 2003. doi: 10.1016/S01429418(02)00118-6.

[3] H. Knidri, R. Belaabed, R.E. Khalfaouy, A. Laajeb, A. Addaou, and A. Lahsini, "Physicochemical characterization of chitin and chitosan producted from parapenaeus longirostris shrimp shell wastes," Journal of Materials and Environmental Science, Vol. 8, No. 10, pp. 3648-3653, 2017.

[4] E.A. Hefian, M.M. Nasef, and A.H. Yahaya, "The preparation and characterization of chitosan/poly (vinyl alcohol) blended films," E-Journal of Chemistry, Vol. 7, No. 4, pp. 1212-1219, 2010. doi: 10.1155/2010/626235

[5] M.G. Cascone, "Dynamic-mechanical properties of bioartificial polymeric materials," Polymer International, Vol. 43, No. 1, pp. 55-69, 1997.

[6] R. Fukae, T. Yamamoto, O. Sangen, T. Saso, T. Kako, and M. Kamachi, "Dynamic mechanical behaviour of poly (vinyl alcohol) film with high syndiotacticity," Polymer Journal, Vol. 22, No. 7, pp. 636-637, 1990. Doi: 10.1295/polymj.22.636

[7] A.T. Htwe, Studies on the Preparation and Characterization of Chitosan-polyvinyl Alcohol Blended Film, Thesis (Master's), Department of Chemistry, University of Yangon, Yangon, Myanmar, 2007.

[8] S.B. Bahrami, S.S. Kordestani, H. Mirzadeh, and P. Mansoori, "Polyvinyl alcohol-chitosan blends: preparation, mechanical and physical properties," Iranian Polymer Journal, Vol. 12, No. 2, pp. 139-146, 2003.

[9] B.D. Mistry, A Handbook of Spectroscopic Data Chemistry (UV, IR, PMR, ${ }^{13} C N M R$ and Mass Spectroscopy), Oxford Book Company, India, pp. 26-63, 2009.

[10] K. Nakamoto, Infrared Spectra of Inorganic and Coordination Compound, $2^{\text {nd }}$ Edition, John Wiley and Sons, New York, United States, 1970. 